\title{
X-RAY AND UV MONITORING OF THE SEYFERT 1.5 GALAXY MARKARIAN 817
}

\author{
Anthony M. Morales ${ }^{1}$, Jon M. Miller ${ }^{1}$, Edward M. CACKetT ${ }^{2}$, Mark T. Reynolds $^{1}$, And Abderahmen Zoghbi ${ }^{1}$
}

\begin{abstract}
We report the results of long-term simultaneous X-ray and UV monitoring of the nearby $(z=0.03145)$ Seyfert 1.5 galaxy Mrk 817 using the Neil Gehrels Swift Observatory XRT and UVOT. Prior work has revealed that the X-ray flux from Mrk 817 has increased by a factor of 40 over the last 40 years, whereas the UV emission has changed by a factor of 2.3. The X-ray emission of Mrk 817 now compares to some of the brightest Seyferts, but it has been poorly studied in comparison. We find that the X-ray $(0.3-10.0 \mathrm{keV})$ and the UVM2 (roughly 2000$2500 \AA$ ) fluxes have fractional variability amplitudes of 0.35 and 0.18 , respectively, over the entire monitoring period (2017 Jan. 2 to 2018 Apr. 20). A cross-correlation analysis is performed on the X-ray (0.3-10.0 keV) and UVM2 light curves over the entire monitoring period, a period of less frequent monitoring (2017 Jan. 2 to 2017 Dec. 11), and a period of more frequent monitoring (2018 Jan. 12 to $2018 \mathrm{Apr}$. 20). The analysis reveals no significant correlation between the two at any given lag for all monitoring periods. Especially given that reverberation studies have found significant lags between optical/UV continuum bands and broad optical lines in Mrk 817, the lack of a significant X-ray-UV correlation may point to additional complexities in the inner or intermediate disk. Mechanical (e.g.,a funnel in the inner disk) and/or relativistic beaming of the X-ray emission could potentially explain the lack of a correlation. Alternatively, scattering in an equatorial wind could also diminish the ability of more isotropic X-ray emission to heat the disk itself.
\end{abstract}

Keywords: accretion, accretion disks — galaxies: active — galaxies: individual (Mrk 817) — galaxies: Seyfert — X-rays: galaxies — X-rays: individual (Mrk 817)

\section{INTRODUCTION}

Active galactic nuclei (AGN) are powered by accretion onto a supermassive black hole. At high fractions of the Eddington limit, this process is mediated by an accretion disk that peaks in the UV. A "corona" is also inferred through X-ray emission in many AGN. Though the corona and accretion disk may be physically distinct, the X-ray and UV emission from these regions may interact with each other. On long timescales (weeks to months), variations in the mass accretion rate through the disk should cause changes in the rate of viscous dissipation, and thereby the UV flux; Compton upscattering in the corona may then drive variations in the X-ray flux. On shorter timescales (less than 1-2 days), rapid variability in the corona - perhaps due to magnetic activity - can drive X-ray flux changes that are reprocessed into UV emission in the disk. On both long and short timescales, then, a very simple picture predicts clear lags and correlations.

Recent research into X-ray and UV variability has produced mixed results. Several teams looking at different sources have found cases where the X-ray emission leads the UV emission (e.g. McHardy et al. 2014; Troyer et al. 2016; Edelson et al. 2015; Edelson et al. 2017; McHardy et al. 2018; Pal and Naik 2018), consistent with X-ray reprocessing. These lags are longer than predicted, though, and they are attributed to larger accretion disks or extra reprocessing mechanisms. In the case of NGC 5548, the X-ray emission has been found to lead the optical emission on timescales of 1 year (Uttley et al. 2003), but it has been noted recently that the X-ray and UV/optical emissions correlate poorly on shorter timescales, which again require extra reprocessing mechanisms to explain such results (Edelson et al. 2015; Gardner \& Done 2017;

\footnotetext{
${ }^{1}$ Department of Astronomy, University of Michigan, 1085 South University Avenue, Ann Arbor, MI 48109-1107, USA, ammoral@umich.edu

${ }^{2}$ Department of Physics \& Astronomy, Wayne State University, 666 W. Hancock Street, Detroit, MI, 48201, USA
}

Starkey et al. 2017). Others, however, have found no correlation between the X-ray and UV emission, attributing this to light bending of a centrally compact X-ray source near to the black hole, X-ray flux in our line of sight not being correlated with the X-ray emission that is reprocessed in the disk, and/or other mechanisms driving the observed UV variability (Robertson et al. 2015; Buisson et al. 2018).

It has also been previously noted that the appearance of significant lags can change depending on the interval or time scale being studied. For example, Gallo et al. (2018) examined X-ray and UV light curves of Mrk 335 and found no correlation between the two, over an eleven year span. However, when they restricted their analysis to only the 8th year of monitoring, they found a highly significant positive correlation of the X-ray leading the UV. They attributed this correlation to a giant X-ray flare that occurred during their monitoring. This and the examples above reveal the complexity of X-ray/UV radiation and the need for a larger number of multi-wavelength studies to better understand disks and diskcorona connections in AGN.

In this paper, we examine the relationship between X-ray and UV variability of the nearby $(z=0.03145)$ Seyfert 1.5 galaxy Mrk $817\left(\log \left(M_{B H} / M_{\odot}\right)=7.586\right.$; Bentz \& Katz 2015). The X-ray emission of Mrk 817 has changed drastically since its discovery decades ago. Previously, the modest X-ray flux of Mrk 817 made it largely inaccessible. However, by 2009, its X-ray flux had increased by a factor of $\sim 40$ since 1990 (Winter et al. 2011), making Mrk 817 one of the brightest Seyferts in the X-ray regime. In contrast, the UV emission only varied by a factor of $\sim 2.3$ over a 30 year period (Winter et al. 2011). The fact that the X-ray light curve has previously shown marked variability while the UV light curve does not, makes Mrk 817 a particularly intriguing AGN. Yet, in comparison to other X-ray-bright Seyfert galaxies, Mrk 817 has been overlooked.

The most recent X-ray spectral study of Mrk 817, by Winter 


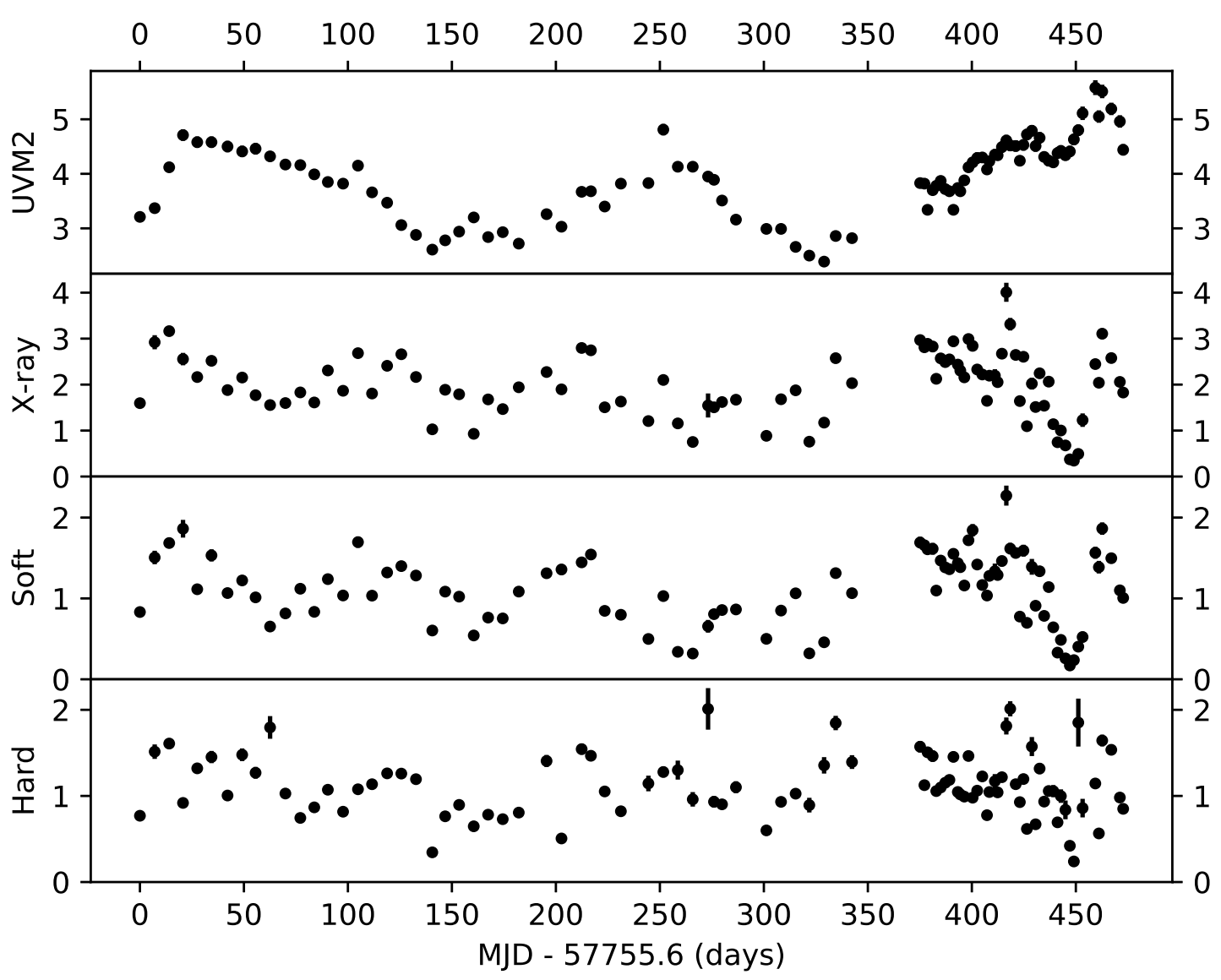

Figure 1. The Swift UVOT/UVM2 flux density $\left(10^{-14} \mathrm{erg} \mathrm{s}^{-1} \mathrm{~cm}^{-2} \AA^{-1}\right)$, XRT X-ray (0.3-10.0 keV) flux (10-11 $\left.\mathrm{erg} \mathrm{s}^{-1} \mathrm{~cm}^{-2}\right), \mathrm{XRT} \mathrm{soft} \mathrm{X-ray}(0.3-2.0 \mathrm{keV})$ flux $\left(10^{-11} \mathrm{erg} \mathrm{s}^{-1} \mathrm{~cm}^{-2}\right)$, and XRT hard X-ray (2.0-10.0 keV) flux $\left(10^{-11} \mathrm{erg} \mathrm{s}^{-1} \mathrm{~cm}^{-2}\right)$ of Mrk 817 are shown from top to bottom, respectively. Most error bars are too small to be visible. The UVM2 flux density shown is the corrected flux density, which was calculated using UVOTSOURCE. The X-ray (0.3-10.0 keV) flux is calculated using a power-law model in XSPEC, while the soft and hard X-ray fluxes are calculated using a broken power-law model with break energy 2.0 $\mathrm{keV}$ in XSPEC. See Section 2 for more details.

et al. (2011), used the six X-ray observations from Swift and single XMM-Newton exposure that were available at the time. They found that the optical/UV fluxes were similar and the optical-X-ray SED changes between observations were due to variations in the X-ray luminosity/spectral shape, concluding that the UV and X-ray are not correlated. Winter et al. (2011) also reported a strong positive correlation between the X-ray spectral slope and X-ray luminosity. We re-examine these results more thoroughly using a cross-correlation analysis on 94 new X-ray and UV observations from Swift . In Section 2, we describe the observations and data reduction. Section 3 details and provides results of a cross-correlation analysis of the $\mathrm{X}$-ray and UV light curves. We end with a discussion of our results in Section 4.

\section{OBSERVATIONS AND DATA REDUCTION}

Mrk 817 was monitored simultaneously using the Swift XRT (Burrows et al. 2005) and the Swift UVOT (Roming et al. 2005) from 2017 Jan. 2 to 2018 Apr. 20. Over this 473-day period, 94 observations were considered, separated in time by an average of $\sim 5.1$ days.

The XRT exposures were taken in "photon counting" mode with a $23.6 \times 23.6$ arcmin FOV. Source events were extracted from a circle with a radius 20 pixels, centered on coordi- nates provided in $\mathrm{NED}^{3}$. Background events were extracted using a circular region of radius 20 pixels, off-center from the source coordinates and outside the source region. Ancillary response files were created for each observation using XRTMKARF, each of which included the provided exposure map to account for bad CCD pixels and columns. Using GRP$\mathrm{PHA}$, the spectra were grouped such that there are at least 10 counts in each bin.

The extracted spectra were then fitted with a power-law model over the $0.3-10.0 \mathrm{keV}$ energy range (mean count rate of $0.5466 \pm 0.0025 \mathrm{cts} \mathrm{s}^{-1}$ ) using XSPEC V.12.9.1P. An absorbed power-law model is not considered, as the galactic HI column density is too small $\left(\mathrm{N}_{\mathrm{H}}=1.50 \times 10^{20} \mathrm{~cm}^{-2}\right.$, Dickey \& Lockman 1990) to produce any discernible effects within the short exposures. For each spectrum, the photon index and normalization, and the corresponding $1 \sigma$ errors on these parameters, were recorded. The flux in the fitting band was obtained using the "flux" command within XSPEC; flux errors were calculated by assuming that the flux has the same fractional error as the power-law normalization. We find that this gives a more conservative estimation of error than the one provided by XSPEC. The X-ray light curve can be seen in the panel

\footnotetext{
3 The NASA/IPAC Extragalactic Database (NED) is operated by the Jet Propulsion Laboratory, California Institute of Technology, under contract with the National Aeronautics and Space Administration.
} 
labeled "X-ray" of Figure 1.

We separately modeled all X-ray spectra using a broken power-law model, in an attempt to allow for a physically distinct "soft excess". The break energy was fixed at $2 \mathrm{keV}$ in each observation, and the flux for the soft $(0.3-2.0 \mathrm{keV})$ and hard $(2.0-10.0 \mathrm{keV}) \mathrm{X}$-rays were recorded. The flux errors were calculated in the same manner as before. The soft and hard X-ray light curves are shown in the panels labeled "Soft" and "Hard" of Figure 1, respectively.

The UV images were taken with the UVOT/UVM2 filter, which has the smallest red-leak of the Swift UVOT UV filters. The UVM2 filter has a central wavelength of $2246 \AA$, an effective wavelength of $2231 \AA$, and a FWHM of $498 \AA$ (Poole et al. 2008). Source events were extracted from the images using a circular region of radius 5 arc-seconds centered on the source. Background emission was accounted for using an annulus ( 33 arc-second inner-radius, 43 arc-second outer-radius) centered on the the source. We calculated the flux density of the UVM2 images using the FTOOLS function UVOTSOURCE. It should be noted that there can be coincidence losses due to the brightness of the source, and UVOTSOURCE provides a reconstructed "true" flux density; this is the flux density we recorded for each observation. The error on each flux density value was calculated as $\sqrt{\text { stat-err }^{2}+\text { sys-err }^{2}}$, where stat-err and sys-err are the statistical error and systematic error on the flux density, respectively. The light curve for the UVM2 is given in the panel labeled "UVM2" of Figure 1. The mean corrected count rate for the entire monitoring period is $46.66 \pm 0.11 \mathrm{cts} \mathrm{s}^{-1}$ with a mean corrected count rate factor of $1.627 \pm 0.004$. It is well-known that UV "dropouts" can occur due to suspect regions of the detector (e.g. Edelson et al. 2015; Edelson et al. 2017). We do not account for "dropouts" here, as the UVM2 light curve has very few obvious points that demonstrate such features.

Light curve data and exposure times for each of the $94 \mathrm{ob}-$ servations is provided in Table 1.

\section{ANALYSIS \& RESULTS}

\subsection{Photon Index/X-ray Relationship}

A plot of the power-law photon index and corresponding $\mathrm{X}$-ray $(0.3-10.0 \mathrm{keV})$ flux is provided in Figure 2. The mean power-law photon index is $2.0047 \pm 0.0117$. We calculated a Spearman correlation coefficient of $r=0.34$ with a two-sided p-value of $p=9.6 \times 10^{-4}$. In contrast, Winter et al. (2011) found a positive correlation between the photon index and $\mathrm{X}$-ray luminosity, with a Spearman correlation coefficient of $r=0.77$ and a two-sided $p$-value of $p=0.05$. Our analysis includes a much larger set of observations, and may therefore more accurately reflect the degree to which the power-law properties are correlated. Combining our points with the data presented in Winter et al. (2011) slightly increases the correlation we originally calculated: $r=0.38$ with $p=1.1 \times 10^{-4}$.

We also provide plots for the soft $X$-ray photon index versus the soft X-ray flux and the hard photon index versus the hard $\mathrm{X}$-ray flux in Figure 3. The soft photon index and hard photon index correspond to the $0.3-2.0 \mathrm{keV}$ and $2.0-10.0 \mathrm{keV}$ energy ranges of the broken power-law model, respectively. The mean soft photon index and mean hard photon index are $2.142 \pm 0.015$ and $1.601 \pm 0.032$, respectively. The soft photon index and soft X-ray flux give a Spearman correlation coefficient of $r=0.12$ with two-sided p-value of $p=0.24$. The hard photon index and hard X-ray flux produce a Spearman correlation coefficient of $r=0.25$ with two-sided p-value of

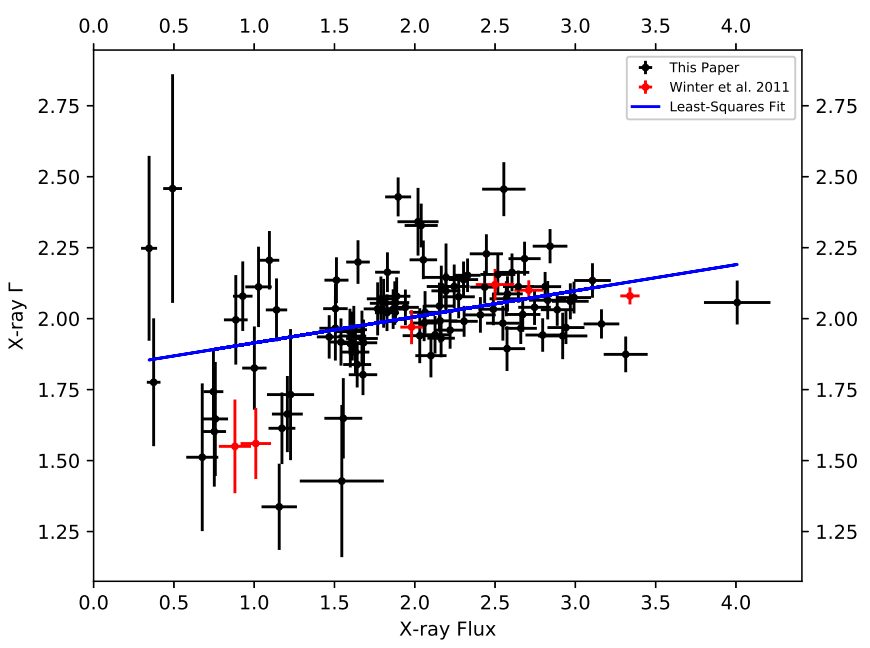

Figure 2. The power-law photon index versus the X-ray (0.3-10.0 keV) flux $\left(10^{-11} \mathrm{erg} \mathrm{s}^{-1} \mathrm{~cm}^{-2}\right)$. The data first presented in our analysis are shown in black; data reported by Winter et al. (2011) are shown in red. The plot has a Spearman correlation coefficient of $r=0.34$ with a two-sided p-value of $p=9.6 \times 10^{-4}$. The mean power-law photon index is $2.0047 \pm 0.0117$. The least-squares linear fit $y=m x+b$ gives $m=0.092, b=1.823$ with squared correlation coefficient $R^{2}=0.111$. See Section 3.1 for more details.

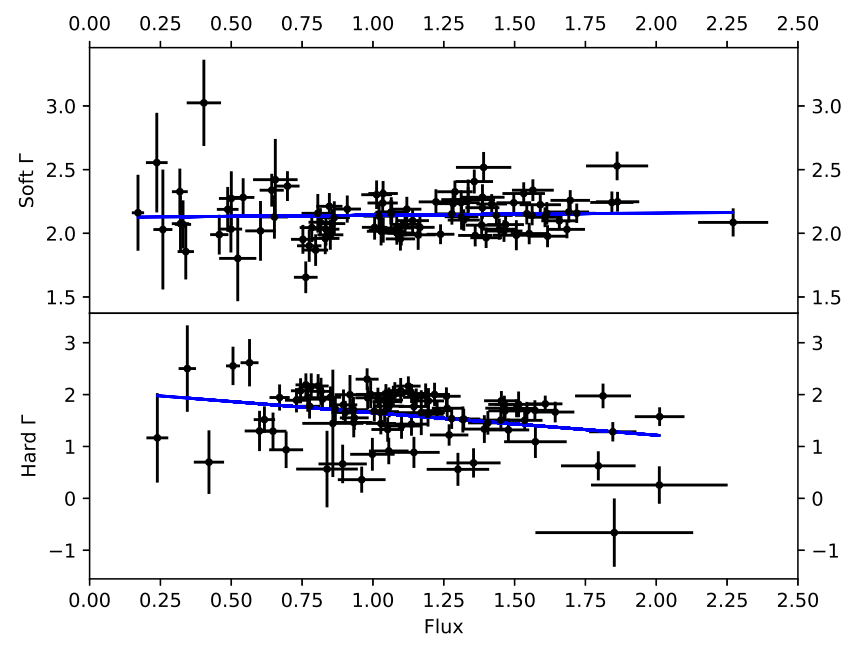

Figure 3. Top Pannel: The broken power-law soft photon index versus soft $\mathrm{X}$-ray flux $\left(10^{-11} \mathrm{erg} \mathrm{s}^{-1} \mathrm{~cm}^{-2}\right)$. The data give a Spearman correlation coefficient of $r=0.12$ with a two-sided p-value of $p=0.24$. The mean soft photon index is $2.143 \pm 0.015$. The least-squares linear fit gives $m=0.017, b=2.124$ with squared correlation coefficient $R^{2}=0.042$. Bottom Panel: The broken power-law hard photon index versus the hard X-ray flux $\left(10^{-11} \mathrm{erg} \mathrm{s}^{-1} \mathrm{~cm}^{-2}\right)$. The data give a Spearman correlation coefficient of $r=0.25$ with a two-sided $\mathrm{p}$-value of $p=0.013$. The mean hard photon index is $1.601 \pm 0.032$. The least-squares linear fit gives $m=-0.433, b=2.082$ with squared correlation coefficient $R^{2}=0.086$. See Section 3.1 for more details.

$p=0.013$.

\subsection{X-ray/UV Correlation Analysis}

A plot (Figure 4) of the X-ray (0.3-10.0 keV) flux versus the UVM2 flux shows minimal correlation between the two bands. Indeed, an analysis of the two produces a Pearson correlation coefficient of $r=0.11$ with a two-sided p-value of $p=0.29$.

A cross-correlation analysis was performed in order to test for correlations at different lags. We use the discrete correlation function (DCF; Edelson \& Krolik 1988, Robertson et al. 


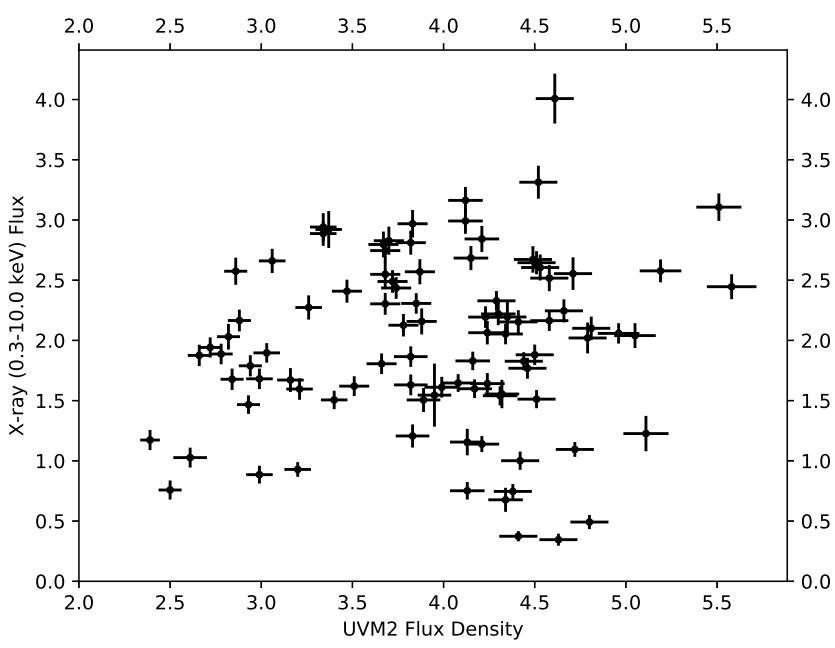

Figure 4. The $\mathrm{X}$-ray $(0.3-10.0 \mathrm{keV})$ flux $\left(10^{-11} \mathrm{erg} \mathrm{s}^{-1} \mathrm{~cm}^{-2}\right)$ versus the UVM2 flux density $\left(10^{-14} \mathrm{erg} \mathrm{s}^{-1} \mathrm{~cm}^{-2} \AA^{-1}\right)$. We calculate a Pearson correlation coefficient of $r=0.11$ with two-sided p-value of $p=0.29$. See Section 3.2 for more details.

2015), because of the uneven sampling of the X-ray and UV observations. A positive value on the lag axis corresponds to the X-ray flux leading the UV flux. The DCF requires the lag time axis to be separated into bins of equal width, $\delta \tau$, which is a free variable. By the definition of the DCF, these bins must contain at least one data point, and - if errors are to be calculated - each bin must contain at least two data points. Choosing a value for $\delta \tau$ is a tradeoff between statistical accuracy and resolution; the former requires larger $\delta \tau$ and the latter requires smaller $\delta \tau$. We set $\delta \tau=50$ days, as this allowed for at least 20 points in each bin, providing reasonable statistical accuracy and a DCF with discernible details.

We calculated 95 and 99 percent confidence curves to evaluate the significance of various potential lags. We first simulated $1000 \mathrm{X}$-ray light curves using the method of Timmer \& König (1995). This procedure requires knowledge of the power spectral density (PSD), which we estimate to be a power-law with a slope of -1.1 and a normalization value of 0.011; this estimation follows the method of Zoghbi et al. (2013). Values are then selected from each simulated light curve such that they correspond to the times at which the real X-ray observations were recorded. The DCF between these simulated values and the real UV light curve is calculated. This produces a distribution of 1000 correlation values at each lag, which we then used to calculate 95 and 99 percent confidence values.

We report no correlation above a $95 \%$ confidence value at any given lag, as can be seen in the first panel of Figure 5. However, via visual inspection, there appears to be an anti-correlation between the X-ray and the UV light curves from 2018 Jan. 12 to 2018 Apr. 20 (MJD 58130 to MJD 58228), a time period of approximately 98 days in which the observation cadence increases. The DCF plot shows an anticorrelation at a lag of $\sim 18.3$ days (see Figure 6), significant at the $95 \%$ level of confidence, corresponding to the X-ray flux leading the UV flux. Owing to this modest significance, the lack of a clear physical interpretation for an anti-correlation, and the robust nature of the $\mathrm{DCF}^{4}$, this putative signature must be regarded skeptically. The light curves were cross-

\footnotetext{
4 This robust nature refers to the method to calculate the DCF as an approximation to a true cross-correlation function.
}

correlated for a period of less frequent observation (2017 Jan. 2 to 2017 Dec. 122, MJD 57755 to MJD 58098), and no significant correlation was found.

The soft and hard X-ray bands were each cross-correlated with the UVM2 as well; these DCFs are plotted in the second and third panels of Figure 5, respectively. The soft X-ray band produces a DCF similar to the full X-ray band (0.3-10.0 $\mathrm{keV}$ ), showing no correlation. The DCF of the hard X-ray band with the UVM2, however, produces a $99 \%$ confident anti-correlation at a lag of $\sim 284$ days, meaning the X-ray leads the UV by $\sim 284$ days. The fact that the lag time scale is comparable to the period over which the monitoring was conducted indicates that this signal should be regarded cautiously. Horne et al. (2004) suggest that the length of the monitoring period should be at least 3 times longer than the lags of interest, whereas Welsh (1999) recommends a monitoring period of at least 4 times longer, but preferably $\sim 10$ times longer, than the lags of interest. In this paper, we follow the suggestion of Horne et al. (2004).

A cross-correlation of the hard and soft X-ray light curves reveals a $99 \%$ significant anti-correlation at a lag of $\sim 235$ days, corresponding to the hard X-ray flux leading the soft Xray flux (see the fourth panel of Figure 5). There also exists $95 \%$ confident positive correlations at lags of $\sim 144$ days and $\sim 349$ days. These positive correlations may also be spurious: the lags are not highly significant, and the robust nature of the DCF can produce such results. Here again, the longest lags are also close to the full period of over which the monitoring was conducted. Visually inspecting the soft and hard X-ray light curves, it appears that the two light curves are tracking each other. We find that the hard and soft X-ray light curves are indeed correlated at lag $\tau=0$ by calculating a Pearson correlation coefficient of $r=0.464$ with a two-sided p-value of $p=2.49 \times 10^{-6}$, corresponding to a $>99 \%$ significance. The disagreement at $\tau=0$ between the DCF and the Pearson coefficient is due to the binning requirement of the DCF.

\section{DISCUSSION}

We have undertaken an extensive X-ray and UV monitoring campaign to better understand Mrk 817, a bright, nearby, Seyfert-1.5 AGN. Although the source is now as bright as the best-known Seyferts, it was much fainter in past decades, and as a result it has not been studied extensively. We crosscorrelated the X-ray and UV light curves using the standard DCF; separate cross-correlations were undertaken for the soft and hard X-ray flux, in recognition of potentially distinct components within the spectrum. We do not find any lag signals at a high level of statistical significance. In this section, we offer plausible physical reasons why strong correlations might be absent.

Many similar analyses have found significant short lags, with the X-rays leading the UV in a manner broadly consistent with reprocessing (McHardy et al. 2014; Troyer et al. 2016; Edelson et al. 2015; Edelson et al. 2017; McHardy et al. 2018; Pal and Naik 2018). In some cases, the observed lags are slightly longer than anticipated by standard thin disk models; this may suggest that AGN disks do not follow such prescriptions or the lags are contaminated by the continuum emission from the broad-line region (Korista \& Goad 2001; Cackett et al. 2018). In the case of Mrk 817, however, the DCFs that we calculated find no robust correlations above the 95\% confidence between the X-ray (0.3-10.0 keV) and UV light curves. Putative lag signals on long time scales - noted above - are too close to the duration of the monitoring to be 


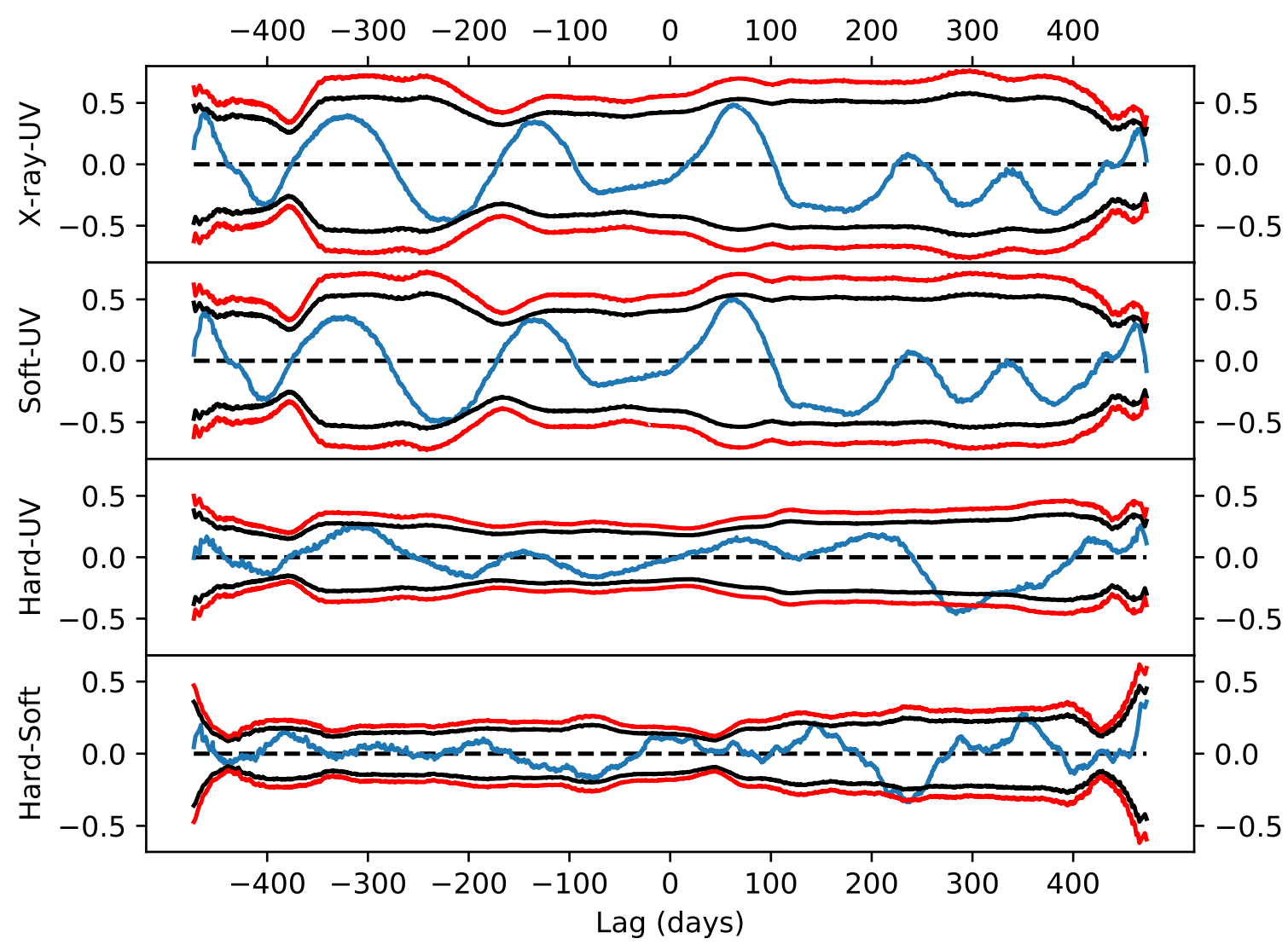

Figure 5. The various calculated DCFs. The black and red curves represent 95 and 99 percent confidence curves, respectively. There is a lack of correlation in the X-ray-UV DCF and the soft X-ray-UV DCF, as all values are less than 95\% confident. A 99\% significant anti-correlation exists in the hard X-ray-UV DCF at a lag of $\sim 284$ days, corresponding to the hard X-ray flux leading the UV flux by $\sim 284$ days. The DCF between the hard and soft X-rays also has a $99 \%$ significant anti- correlation at $\sim 235$ days, corresponding to the hard X-ray flux leading the soft X-ray flux. See Section 3.2 for calculation details and Section 4 for a discussion of the results.

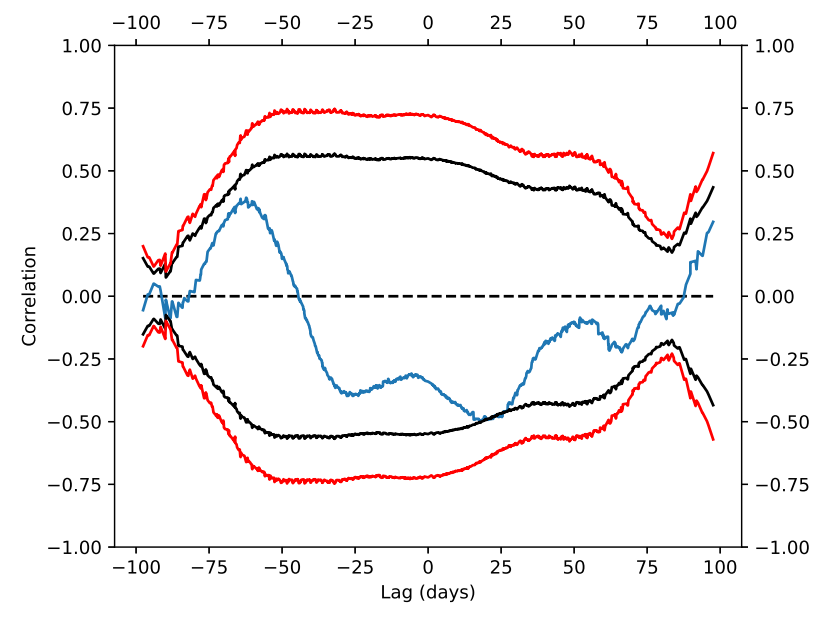

Figure 6. The DCF between the higher cadence portions of the X-ray (0.3-10.0 keV) and UVM2 light curves is shown. A 95\% significant anticorrelation exists at a lag of $\sim 18.3$ days, corresponding to the X-ray leading the UV. See Section 3.2 for more details.

credible. Though uncommon, non-correlations have been reported recently (Robertson et al. 2015; Buisson et al. 2018). This lack of a significant X-ray/UV correlation is inconsistent with the optical variability of Mrk 817, as it has been reverberation mapped, i.e. lags have been calculated between the $\mathrm{H} \beta$ and continuum light curves (Peterson et al 1998; Denney et al. 2010). This suggests that, in this source, the X-ray variability is not the primary driver of photoionization and line variability in the BLR. This is at odds with the standard lamppost model whereby a small, centrally located X-ray source drives variability in the UV/optical continuum and emission lines.

Geometric beaming - or perhaps "funneling" - of the Xray emission represents one potential means by which reprocessing of X-ray emission into UV flux might be prevented. For an AGN with a high-Eddington fraction, for instance, the inner accretion disk may fail to cool efficiently, giving it an increased scale height above the local surface of the disk. This geometry may serve as an inner funnel that could block the X-ray emission from larger disk radii. This explanation requires that we have a privileged viewing angle into the funnel. Moreover, Mrk 817 has a low-Eddington fraction: $L_{\text {bol }} / L_{\text {Edd }}=0.1950\left(\log L_{\text {bol }}=44.99\right.$, Woo \& Urry 2002). Recent work on NGC 5548 and NGC 4151, in particular, has suggested that additional geometries may be needed to account for X-ray and UV correlations (e.g., Edelson et al. 2017; Gardner \& Done 2017). Moreover, X-ray observations of NGC 4151 find evidence of a warp or bulge in the inner accretion disk (Miller et al. 2018; Zoghbi et al. 2018) though its Eddington fraction is even lower than that of Mrk 817.

A qualitatively distinct but physically similar scenario is that the X-ray flux is relativistically beamed away from the disk, owing to bulk motion normal to the disk. If the X-ray 
corona is the base of a jet (Markoff, Nowak, \& Wilms 2005; Miller et al. 2006), where acceleration away from the disk may occur, then the velocities required to escape the regions closest to the black hole may serve to naturally beam the $\mathrm{X}$ ray emission away from the disk. Like most other Seyferts, however, Mrk 817 is not radio-loud, so if this process is at work the corona might be the base of a "failed" jet.

It is also possible that a dense outflow from the inner disk could inhibit the reprocessing of X-rays into UV flux in the disk. A disk wind that covers the inner accretion disk might scatter a fraction of the X-ray emission before this flux reaches the optically-thick accretion disk. Winter et al. (2011) find no evidence of X-ray absorption consistent with an out- flow; however, disk winds - especially in the inner disk - may be primarily equatorial. If we observe Mrk 817 at a low inclination (closer to the pole), a wind may operate but not be detected. Recent observations of NGC 5548 have also revealed evidence of dense, transient outflows from the inner disk (Kaastra et al. 2014). Additional X-ray and UV spectroscopy of Mrk 817 may reveal if the source was in a similar state during the period of our monitoring, and/or when Winter et al. (2011) studied the source with XMM-Newton. Obscuration by transient clouds from a warm absorber and a neutral absorber can mask the intrinsic variability of the X-ray emission, which could lead to a lack of correlation between the $\mathrm{X}$-ray and UV emission.

\section{REFERENCES}

Bentz, M. C., \& Katz, S. 2015, PASP, 127, 67

Buisson, D. J. K., et al. 2018, MNRAS, 475, 2306

Burrows, D. N., et al. 2005, Sp. Sci. Rev., 120, 165

Cackett, E. M., et al. 2018, ApJ, 857, 53

Denney, K. D., Peterson, B. M., Pogge, R. W., et al. 2010, ApJ, 721, 715

Dickey, J. M., \& Lockman, F. J. 1990, ARAA, 28, 215

Edelson, R., Gelbord, J., Cackett, E., et al. 2017, ApJ, 840, 41

Edelson, R., Gelbord, J. M., Horne, K., et al. 2015, ApJ, 806, 129

Edelson, R. A., \& Krolik, J. H. 1988, ApJ, 333, 646

Gallo, L. C., et al. 2018, MNRAS, 478, 2557

Gardner, E., \& Done, C. 2017, MNRAS, 470, 3591

Horne, K., Peterson, B. M., Collier, S. J., \& Netzer, H., PASP, 2004, 116, 465

Kaastra, J., Kriss, G., Cappi, M., et al., 2014, Science, 345, 64

Korista, K. T., \& Goad, M. R. 2001, ApJ, 553, 695

Markoff, S., Nowak, M. A., \& Wilms, J. 2005, ApJ, 635, 1203

McHardy, I. M., et al. 2014, MNRAS, 444, 1469

McHardy, I. M., et al. 2018, MNRAS, 480, 2881
Miller, J. M., Cackett, E., Zoghbi, A., et al. 2018, ApJ, 865, 97

Miller, J. M., Homan, J., Steeghs, D., et al. 2006, ApJ, 653, 525

Pal, M., \& Naik, S. 2018, MNRAS, 474, 5351

Peterson, B. M., Wanders, I., Bertram, R., et al. 1998, ApJ, 501, 82

Poole, T. S., et al. 2008, MNRAS, 383, 627

Robertson, D. R. S., et al. 2015, MNRAS, 453, 3455

Roming, P. W. A., et al. 2005, Sp. Sci. Rev., 120, 95

Starkey, D., Horne, K., Fausnaugh, M. M., et al. 2017, ApJ 835, 65

Timmer, J., \& König M. 1995, A\&A, 300, 707

Troyer, J., et al. 2016, MNRAS, 456, 4040

Uttley, P., Edelson, R., McHardy, I. M., et al. 2003, ApJL, 584, L53

Vaughan, S., et al. 2003, MNRAS, 345, 1271

Welsh, W. F. 1999, PASP, 111, 1347

Winter, L. M., Danforth, C., Vasudevan, R., et al. 2011, ApJ, 728, 28

Woo, J.-H., \& Urry, C. M. 2002, ApJ, 579, 530

Zoghbi, A., Miller, J. M., Cackett, E. M., et al., 2018, ApJ, in prep.

Zoghbi, A., Reynolds, C., \& Cackett, E. M. 2013, ApJ, 777, 24 
Table 1 Data. The observation I.D. (denoted OBSID), start time in MJD, exposure time in seconds, UVM2 flux density and flux density error $\left(10^{-14} \mathrm{erg} \mathrm{s}^{-1} \mathrm{~cm}^{-2} \AA^{-1}\right)$, X-ray $(0.3-10.0 \mathrm{keV})$, soft X-ray $(0.3-2.0 \mathrm{keV})$, and hard X-ray $(2.0-10.0 \mathrm{keV})$ fluxes and flux errors $\left(10^{-11} \mathrm{erg} \mathrm{s}^{-1} \mathrm{~cm}^{-2}\right)$ for each observation are listed. Start time MJD 57755.6 is 2017 Jan. 02 at 14:24:00.000 UTC, and end time MJD 58228.4 is 2018 Apr. 20 at 09:36:00.000 UTC.

\begin{tabular}{|c|c|c|c|c|c|c|c|c|c|c|}
\hline OBSID & Start Time & Exposure Time & UVM2 & UVM2 Error & X-ray & X-ray Error & Soft & Soft Error & Hard & Hard Error \\
\hline 00037592002 & 57755.6 & 981.4 & 3.21 & 0.073 & 1.596 & 0.0893 & 0.832 & 0.0501 & 0.77 & 0.0464 \\
\hline 00037592003 & 57762.7 & 1088.8 & 3.37 & 0.073 & 2.921 & 0.1536 & 1.506 & 0.0835 & 1.514 & 0.0839 \\
\hline 00037592004 & 57769.7 & 996.4 & 4.12 & 0.095 & 3.163 & 0.1111 & 1.685 & 0.0636 & 1.608 & 0.0607 \\
\hline 00037592005 & 57776.3 & 1001.4 & 4.71 & 0.104 & 2.555 & 0.1352 & 1.862 & 0.1096 & 0.919 & 0.0541 \\
\hline 00037592006 & 57783.2 & 1213.7 & 4.58 & 0.104 & 2.164 & 0.0856 & 1.113 & 0.0479 & 1.32 & 0.0568 \\
\hline 00037592007 & 57790.0 & 789.2 & 4.58 & 0.104 & 2.517 & 0.1087 & 1.532 & 0.075 & 1.452 & 0.0711 \\
\hline 00037592008 & 57797.7 & 986.4 & 4.5 & 0.104 & 1.881 & 0.0847 & 1.066 & 0.0523 & 1.005 & 0.0493 \\
\hline 00037592009 & 57804.8 & 949.0 & 4.41 & 0.104 & 2.154 & 0.097 & 1.222 & 0.0608 & 1.478 & 0.0735 \\
\hline 00037592010 & 57811.2 & 941.5 & 4.46 & 0.104 & 1.769 & 0.0867 & 1.013 & 0.0542 & 1.269 & 0.0679 \\
\hline 00037592011 & 57818.2 & 936.5 & 4.32 & 0.095 & 1.555 & 0.1179 & 0.652 & 0.0478 & 1.796 & 0.1316 \\
\hline 00037592012 & 57825.6 & 1071.3 & 4.17 & 0.095 & 1.599 & 0.0774 & 0.815 & 0.042 & 1.028 & 0.053 \\
\hline 00037592013 & 57832.7 & 1121.3 & 4.16 & 0.095 & 1.83 & 0.0768 & 1.12 & 0.0513 & 0.745 & 0.0341 \\
\hline 00037592014 & 57839.5 & 1013.9 & 3.99 & 0.095 & 1.611 & 0.0861 & 0.834 & 0.0476 & 0.867 & 0.0494 \\
\hline 00037592015 & 57846.0 & 1211.2 & 3.85 & 0.082 & 2.307 & 0.0851 & 1.239 & 0.049 & 1.072 & 0.0424 \\
\hline 00037592016 & 57853.3 & 981.4 & 3.82 & 0.092 & 1.865 & 0.0853 & 1.036 & 0.0511 & 0.817 & 0.0403 \\
\hline 00037592017 & 57860.5 & 983.9 & 4.15 & 0.095 & 2.684 & 0.1 & 1.696 & 0.0697 & 1.077 & 0.0443 \\
\hline 00037592018 & 57867.2 & 906.5 & 3.66 & 0.082 & 1.806 & 0.0858 & 1.034 & 0.0535 & 1.136 & 0.0588 \\
\hline 00037592019 & 57874.5 & 976.4 & 3.47 & 0.082 & 2.409 & 0.0955 & 1.321 & 0.0567 & 1.262 & 0.0542 \\
\hline 00037592020 & 57881.3 & 1023.9 & 3.06 & 0.073 & 2.66 & 0.1003 & 1.4 & 0.0557 & 1.259 & 0.0501 \\
\hline 00037592021 & 57888.4 & 991.4 & 2.88 & 0.063 & 2.165 & 0.0906 & 1.283 & 0.0595 & 1.194 & 0.0554 \\
\hline 00037592022 & 57896.2 & 581.9 & 2.61 & 0.092 & 1.027 & 0.0823 & 0.603 & 0.0538 & 0.345 & 0.0308 \\
\hline 00037592023 & 57902.4 & 946.5 & 2.78 & 0.063 & 1.887 & 0.0853 & 1.084 & 0.052 & 0.763 & 0.0366 \\
\hline 00037592024 & 57909.1 & 988.9 & 2.94 & 0.063 & 1.789 & 0.0877 & 1.022 & 0.0554 & 0.896 & 0.0486 \\
\hline 00037592025 & 57916.1 & 946.5 & 3.2 & 0.073 & 0.929 & 0.0609 & 0.542 & 0.0397 & 0.647 & 0.0474 \\
\hline 00037592026 & 57923.0 & 981.4 & 2.84 & 0.063 & 1.678 & 0.0885 & 0.763 & 0.0418 & 0.782 & 0.0429 \\
\hline 00037592027 & 57930.1 & 983.9 & 2.93 & 0.063 & 1.467 & 0.077 & 0.753 & 0.0419 & 0.73 & 0.0406 \\
\hline 00037592028 & 57937.8 & 1006.4 & 2.72 & 0.063 & 1.942 & 0.0837 & 1.084 & 0.05 & 0.807 & 0.0372 \\
\hline 00037592030 & 57951.1 & 874.1 & 3.26 & 0.073 & 2.273 & 0.1006 & 1.312 & 0.0652 & 1.406 & 0.0699 \\
\hline 00037592031 & 57958.3 & 966.5 & 3.03 & 0.073 & 1.896 & 0.0808 & 1.358 & 0.0661 & 0.506 & 0.0247 \\
\hline 00037592033 & 57968.0 & 946.5 & 3.67 & 0.082 & 2.796 & 0.1073 & 1.446 & 0.0597 & 1.544 & 0.0637 \\
\hline 00037592034 & 57972.5 & 978.9 & 3.68 & 0.082 & 2.746 & 0.1016 & 1.543 & 0.0623 & 1.466 & 0.0592 \\
\hline 00037592035 & 57979.1 & 998.9 & 3.4 & 0.073 & 1.506 & 0.0764 & 0.846 & 0.0463 & 1.052 & 0.0575 \\
\hline 00037592036 & 57986.9 & 926.5 & 3.82 & 0.092 & 1.631 & 0.0863 & 0.798 & 0.0441 & 0.822 & 0.0455 \\
\hline 00037592038 & 58000.1 & 759.2 & 3.83 & 0.092 & 1.207 & 0.0961 & 0.499 & 0.0396 & 1.145 & 0.0909 \\
\hline 00037592039 & 58007.3 & 949.0 & 4.81 & 0.104 & 2.101 & 0.0972 & 1.029 & 0.0503 & 1.278 & 0.0625 \\
\hline 00037592040 & 58014.2 & 1046.4 & 4.13 & 0.095 & 1.156 & 0.1103 & 0.339 & 0.0288 & 1.3 & 0.1103 \\
\hline 00037592041 & 58021.4 & 1058.8 & 4.13 & 0.095 & 0.752 & 0.0731 & 0.318 & 0.028 & 0.96 & 0.0843 \\
\hline 00037592042 & 58028.8 & 334.6 & 3.95 & 0.092 & 1.546 & 0.2617 & 0.656 & 0.0786 & 2.011 & 0.2412 \\
\hline 00037592043 & 58031.6 & 616.8 & 3.89 & 0.092 & 1.506 & 0.0996 & 0.805 & 0.0573 & 0.932 & 0.0663 \\
\hline 00037592044 & 58035.5 & 996.4 & 3.51 & 0.082 & 1.62 & 0.0821 & 0.856 & 0.0461 & 0.902 & 0.0486 \\
\hline 00037592045 & 58042.2 & 978.9 & 3.16 & 0.073 & 1.671 & 0.099 & 0.864 & 0.0549 & 1.101 & 0.0699 \\
\hline 00037592046 & 58056.8 & 751.7 & 2.99 & 0.073 & 0.885 & 0.074 & 0.5 & 0.0437 & 0.6 & 0.0524 \\
\hline 00037592047 & 58063.9 & 981.4 & 2.99 & 0.073 & 1.681 & 0.085 & 0.85 & 0.0459 & 0.931 & 0.0503 \\
\hline 00037592048 & 58070.9 & 891.5 & 2.66 & 0.063 & 1.875 & 0.0888 & 1.063 & 0.0545 & 1.026 & 0.0526 \\
\hline 00037592049 & 58077.5 & 856.6 & 2.5 & 0.063 & 0.758 & 0.0793 & 0.321 & 0.0306 & 0.893 & 0.0853 \\
\hline 00037592050 & 58084.6 & 1046.4 & 2.39 & 0.054 & 1.173 & 0.0837 & 0.458 & 0.0322 & 1.355 & 0.0955 \\
\hline 00037592051 & 58090.2 & 973.9 & 2.86 & 0.063 & 2.574 & 0.1126 & 1.313 & 0.0594 & 1.847 & 0.0836 \\
\hline 00037592052 & 58098.0 & 869.1 & 2.82 & 0.063 & 2.031 & 0.1066 & 1.065 & 0.0612 & 1.393 & 0.08 \\
\hline 00037592056 & 58130.7 & 981.4 & 3.83 & 0.082 & 2.969 & 0.1143 & 1.691 & 0.0714 & 1.572 & 0.0664 \\
\hline 00037592057 & 58132.8 & 983.9 & 3.82 & 0.082 & 2.812 & 0.1 & 1.658 & 0.0636 & 1.125 & 0.0432 \\
\hline 00037592058 & 58134.3 & 1028.9 & 3.34 & 0.073 & 2.887 & 0.102 & 1.607 & 0.0617 & 1.507 & 0.0579 \\
\hline 00037592059 & 58136.8 & 794.1 & 3.7 & 0.082 & 2.828 & 0.1166 & 1.614 & 0.0727 & 1.463 & 0.0659 \\
\hline 00037592060 & 58138.5 & 993.9 & 3.78 & 0.082 & 2.126 & 0.0932 & 1.097 & 0.0517 & 1.056 & 0.0498 \\
\hline 00037592061 & 58140.6 & 876.5 & 3.87 & 0.082 & 2.57 & 0.1042 & 1.468 & 0.0647 & 1.098 & 0.0484 \\
\hline 00037592062 & 58142.8 & 1063.8 & 3.72 & 0.082 & 2.489 & 0.094 & 1.384 & 0.0561 & 1.153 & 0.0467 \\
\hline 00037592063 & 58144.8 & 956.5 & 3.68 & 0.082 & 2.549 & 0.1048 & 1.361 & 0.0602 & 1.186 & 0.0524 \\
\hline 00037592064 & 58146.8 & 976.4 & 3.34 & 0.073 & 2.941 & 0.1153 & 1.552 & 0.0652 & 1.453 & 0.0611 \\
\hline 00037592065 & 58148.9 & 1066.3 & 3.74 & 0.082 & 2.435 & 0.0902 & 1.436 & 0.0575 & 1.046 & 0.0419 \\
\hline 00037592066 & 58150.1 & 986.4 & 3.68 & 0.082 & 2.304 & 0.0907 & 1.386 & 0.06 & 1.018 & 0.0441 \\
\hline 00037592067 & 58152.0 & 751.7 & 3.88 & 0.082 & 2.158 & 0.108 & 1.16 & 0.0615 & 0.992 & 0.0526 \\
\hline 00037592068 & 58154.0 & 993.9 & 4.12 & 0.095 & 2.992 & 0.1063 & 1.719 & 0.0668 & 1.464 & 0.0568 \\
\hline 00037592069 & 58156.0 & 814.1 & 4.21 & 0.095 & 2.842 & 0.108 & 1.843 & 0.0763 & 0.979 & 0.0406 \\
\hline 00037592070 & 58158.2 & 1266.1 & 4.29 & 0.104 & 2.329 & 0.0812 & 1.419 & 0.0539 & 1.061 & 0.0403 \\
\hline 00037592071 & 58160.6 & 1071.3 & 4.3 & 0.095 & 2.22 & 0.0934 & 1.166 & 0.0519 & 1.227 & 0.0546 \\
\hline 00037592072 & 58162.9 & 1026.4 & 4.08 & 0.095 & 1.646 & 0.0735 & 1.036 & 0.052 & 0.776 & 0.039 \\
\hline 00037592073 & 58164.0 & 971.4 & 4.23 & 0.095 & 2.194 & 0.0901 & 1.279 & 0.0577 & 1.046 & 0.0472 \\
\hline 00037592074 & 58166.7 & 414.6 & 4.35 & 0.104 & 2.195 & 0.1395 & 1.336 & 0.0949 & 1.171 & 0.0831 \\
\hline 00037592075 & 58168.0 & 956.5 & 4.34 & 0.095 & 2.053 & 0.0857 & 1.289 & 0.0626 & 1.041 & 0.0506 \\
\hline 00037592076 & 58170.1 & 968.9 & 4.49 & 0.104 & 2.672 & 0.1102 & 1.463 & 0.0653 & 1.218 & 0.0544 \\
\hline 00037592077 & 58172.2 & 866.6 & 4.61 & 0.104 & 4.008 & 0.207 & 2.271 & 0.1237 & 1.812 & 0.0987 \\
\hline 00037592078 & 58174.1 & 981.4 & 4.52 & 0.104 & 3.313 & 0.1369 & 1.616 & 0.0705 & 2.012 & 0.0877 \\
\hline
\end{tabular}


MoRALES ET AL.

TABLE 1 (continued)

\begin{tabular}{|c|c|c|c|c|c|c|c|c|c|c|}
\hline OBSID & Start Time & Exposure Time & UVM2 & UVM2 Error & X-ray & X-ray Error & Soft & Soft Error & Hard & Hard Error \\
\hline 00037592079 & 58176.7 & 1056.4 & 4.51 & 0.104 & 2.645 & 0.0957 & 1.562 & 0.0608 & 1.136 & 0.0442 \\
\hline 00037592080 & 58178.7 & 981.4 & 4.24 & 0.095 & 1.641 & 0.0872 & 0.775 & 0.0431 & 0.928 & 0.0516 \\
\hline 00037592081 & 58180.4 & 799.1 & 4.53 & 0.104 & 2.605 & 0.1077 & 1.591 & 0.0729 & 1.196 & 0.0548 \\
\hline 00037592082 & 58182.1 & 1013.9 & 4.72 & 0.104 & 1.095 & 0.0614 & 0.698 & 0.0427 & 0.617 & 0.0378 \\
\hline 00037592083 & 58184.5 & 1006.4 & 4.79 & 0.104 & 2.02 & 0.1279 & 1.391 & 0.0977 & 1.573 & 0.1105 \\
\hline 00037592084 & 58186.3 & 1006.4 & 4.51 & 0.104 & 1.512 & 0.0749 & 0.909 & 0.0483 & 0.671 & 0.0356 \\
\hline 00037592085 & 58188.2 & 939.0 & 4.66 & 0.104 & 2.246 & 0.0954 & 1.336 & 0.0636 & 1.318 & 0.0627 \\
\hline 00037592086 & 58190.4 & 1026.4 & 4.31 & 0.095 & 1.541 & 0.0792 & 0.784 & 0.0427 & 0.934 & 0.0509 \\
\hline 00037592087 & 58192.6 & 901.5 & 4.24 & 0.104 & 2.064 & 0.0974 & 1.14 & 0.0576 & 1.057 & 0.0534 \\
\hline 00037592088 & 58194.8 & 998.9 & 4.21 & 0.095 & 1.139 & 0.0665 & 0.643 & 0.0426 & 1.056 & 0.07 \\
\hline 00037592089 & 58196.8 & 1013.9 & 4.38 & 0.104 & 0.746 & 0.0617 & 0.33 & 0.0285 & 0.694 & 0.0599 \\
\hline 00037592090 & 58198.4 & 866.6 & 4.42 & 0.104 & 1.001 & 0.0758 & 0.487 & 0.0383 & 0.998 & 0.0784 \\
\hline 00037592091 & 58200.6 & 1088.8 & 4.34 & 0.095 & 0.677 & 0.0997 & 0.259 & 0.0336 & 0.838 & 0.1089 \\
\hline 00037592092 & 58202.8 & 1018.9 & 4.41 & 0.104 & 0.374 & 0.0425 & 0.171 & 0.0216 & 0.421 & 0.0534 \\
\hline 00037592093 & 58204.7 & 601.9 & 4.63 & 0.104 & 0.345 & 0.0499 & 0.237 & 0.0383 & 0.239 & 0.0386 \\
\hline 00037592094 & 58206.8 & 956.5 & 4.8 & 0.104 & 0.492 & 0.0586 & 0.403 & 0.0606 & 1.852 & 0.2784 \\
\hline 00037592095 & 58208.9 & 292.2 & 5.11 & 0.124 & 1.226 & 0.1468 & 0.523 & 0.0657 & 0.859 & 0.1078 \\
\hline 00037592098 & 58215.0 & 896.5 & 5.58 & 0.136 & 2.446 & 0.1035 & 1.565 & 0.0739 & 1.145 & 0.054 \\
\hline 00037592100 & 58218.3 & 836.6 & 5.51 & 0.124 & 3.107 & 0.1143 & 1.863 & 0.0766 & 1.643 & 0.0675 \\
\hline 00037592101 & 58222.7 & 1106.3 & 5.19 & 0.114 & 2.577 & 0.0951 & 1.497 & 0.0613 & 1.536 & 0.0629 \\
\hline 00037592103 & 58226.8 & 1106.3 & 4.96 & 0.114 & 2.058 & 0.0845 & 1.1 & 0.0488 & 0.981 & 0.0436 \\
\hline 00037592104 & 58228.4 & 1311.1 & 4.44 & 0.104 & 1.827 & 0.075 & 1.006 & 0.0448 & 0.85 & 0.0379 \\
\hline
\end{tabular}

